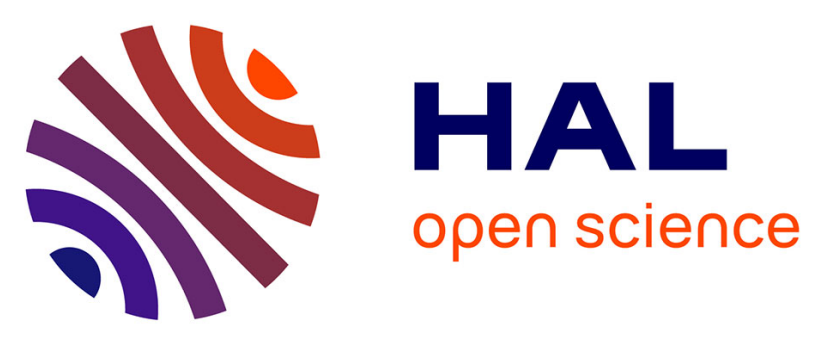

\title{
Numerical and experimental study of a high-power narrow-line phase-locked tapered lasers array in external cavity
}

David Pabœuf, Gaëlle Lucas-Leclin, Patrick Georges, Nicolas Michel, Michel Krakowski, Jun Lim, Slawomir Sujecki, Eric Larkins

\section{To cite this version:}

David Pabœuf, Gaëlle Lucas-Leclin, Patrick Georges, Nicolas Michel, Michel Krakowski, et al.. Numerical and experimental study of a high-power narrow-line phase-locked tapered lasers array in external cavity. Advanced Solid-State Photonics, Feb 2009, Denver, United States. hal-00534744

\section{HAL Id: hal-00534744 \\ https://hal-iogs.archives-ouvertes.fr/hal-00534744}

Submitted on 10 Nov 2010

HAL is a multi-disciplinary open access archive for the deposit and dissemination of scientific research documents, whether they are published or not. The documents may come from teaching and research institutions in France or abroad, or from public or private research centers.
L'archive ouverte pluridisciplinaire HAL, est destinée au dépôt et à la diffusion de documents scientifiques de niveau recherche, publiés ou non, émanant des établissements d'enseignement et de recherche français ou étrangers, des laboratoires publics ou privés. 


\title{
Numerical and experimental study of a high-power narrow- line phase-locked tapered lasers array in external cavity
}

\author{
D. Paboeuf, G. Lucas-Leclin, P. Georges \\ Laboratoire Charles Fabry de l'Institut d'Optique, CNRS, Univ Paris-Sud, Campus Polytechnique, RD128, 91127 Palaiseau - France \\ david.paboeuf@institutoptique.fr \\ N. Michel, M. Krakowski \\ Alcatel-Thales III-V Lab, RD128, 91767 Palaiseau-France \\ J. Lim, S. Sujecki, E. Larkins \\ The School of Electronic and Electrical Engineering, University of Nottingham, University Park, Nottingham NG7 2RD - UK

\begin{abstract}
We describe both coherent combining and wavelength stabilization of 10 tapered lasers in an external Talbot cavity with a volume Bragg grating. 1.7W at $976 \mathrm{~nm}$ in a narrow bandwidth, high coherent beam are obtained.

(C)2008 Optical Society of America

OCIS codes: (140.2010) Diode laser arrays, (140.3298) Laser beam combining, (050.7330) Volume gratings
\end{abstract}

\section{Introduction}

High-power laser diode arrays are very attractive for applications such as solid-state laser pumping or laser material processing due to a very good overall efficiency. Inconveniently, however, their beam quality and spectrum bandwidth are often too far from the requirements for demanding applications. A well-known way to enhance the overall spatial quality of several independent lasers is to induce a coherent combining of their beams by phase locking between the emitters. This has already been investigated successfully with $\mathrm{CO}_{2}$ lasers, fiber lasers and semiconductor diode lasers [1]. In the latter case however the best results to date are limited by the self-operation of the bar. Moreover, volume Bragg gratings are extensively utilized for spectral narrowing and stabilization of broad area lasers and arrays [2]. We propose to take benefit of the self-imaging Talbot properties inside an external cavity to induce the coherent emission of an array of index-guided tapered lasers, and to force them to operate in the inphase spatial supermode. With the aim to favor coherent coupling and to strongly control the emission wavelength, we use a volume Bragg grating (VBG) as the external cavity mirror of the Talbot cavity.

\section{Theoretical study}

The Talbot effect describes the self-imaging properties of a periodic coherent field [3]. It is a pure Fresnel diffractive phenomenon resulting from the free-space propagation of a periodic field. The source array produces exact replica (in amplitude and phase) of the emitted field at integer multiples of the so-called Talbot distance $\left(Z_{T}=2 p^{2} / \lambda\right)$, which is linked to the spatial period $\mathrm{p}$ of the optical field. Within an external cavity designed to precisely reflect one of these replica onto the array, a maximal coupling between adjacent emitters may be obtained, resulting in the coherent emission of the entire bar. Actually for a roundtrip cavity length equal to one Talbot distance, the in-phase and outof-phase modes have identical coupling strengths, which forbids any discrimination between them. On the contrary the spatial discrimination between these 2 modes is maximal after a propagation of $\mathrm{Z}_{\mathrm{T}} / 2$. Indeed at that distance, the out-of-phase mode is self-imaged whereas the in-phase mode is imaged with a lateral shift of $\mathrm{p} / 2$. By placing the external mirror at $Z_{\mathrm{T}} / 2$ with an angle of $\lambda / 2 \mathrm{p}$, the in-phase mode may then be selected.

We have developed a numerical model to describe the behavior of such a Talbot external cavity with a bar of index-guided tapered laser diodes. The electrical and optical description of the tapered emitters has already been studied and is described in [4]. The isothermal electrical model consists of the 1D unipolar carrier-diffusion equation, where the injection current density $\mathrm{J}(\mathrm{x})$ is assumed fixed constant inside the stripe. This equation is used to model the gain distribution and carrier lensing effects. The optical fields in the tapered laser are modeled with the 2D wideangle finite difference beam propagation method (WA-FDBPM) with the effective index approximation. Furthermore the free-space propagation in the external cavity is based on Fresnel diffraction equations, and a plane output mirror $(\mathrm{R}=40 \%)$ has been considered. The optical beam is assumed to be TE polarized and monochromatic. Eventually an iterative approach similar to the Fox-Li method is used in order to compute the array spatial mode. The optical beam is propagated back and forth inside the cavity until it reaches a steady state in terms of power and amplitude profile.

With the help of these simulations, the strong modal discrimination of the Talbot cavity has been confirmed: without tilting the output mirror, the laser array oscillates in the out-of-phase mode whereas with a tilt angle of $\lambda / 2 \mathrm{p}$, 
the in-phase mode is selected. The output power vs the operating current has been computed for the in-phase supermode operation of the laser bar, and compared well to experimental L-I characteristic (Figure 4). Furthermore a map of the intensity repartition inside the semiconductor medium has been simulated in Figure 1 . Due to the asymmetry of the cavity and the finite size of the array, the Talbot replicas are distorted at the edges of the laser array. Thus the optical field reflected by the external mirror is imperfectly coupled into the edge waveguides. This results in extra propagation losses inside the index-guided lasers and reduces the effective external-cavity feedback up to $20 \%$ for off-axis emitters as compared to $30 \%$ for the center one.

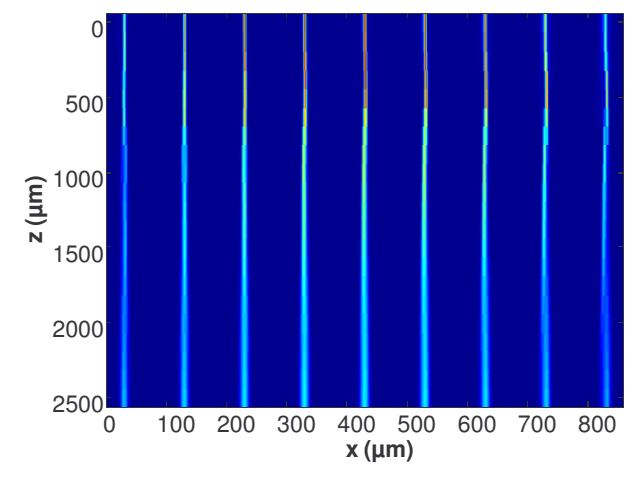

Fig. 1. Intensity map inside the laser array ( $I=4.4 \mathrm{~A}, \mathrm{R}=40 \%, \alpha=\lambda / 2 \mathrm{p})$

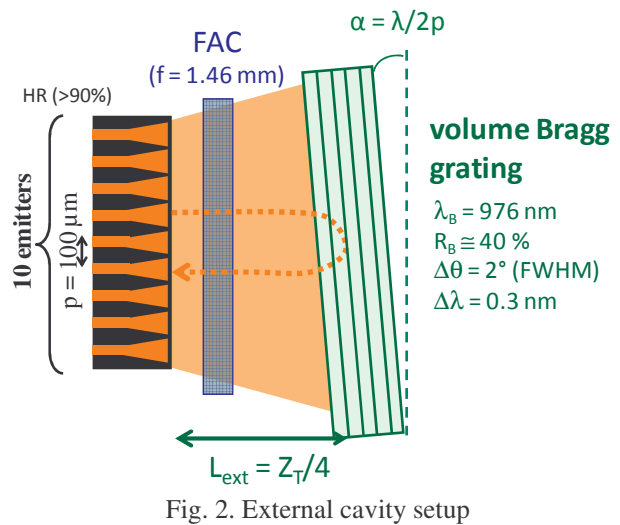

Fig. 2. External cavity setup

\section{Experimental Results}

In our experiments, the laser source is an array of 10 index-guided tapered lasers with a $100 \mu$ m-pitch emitting around $975 \mathrm{~nm}$. The active layer consists of a strained GaInAs quantum well embedded in a large optical cavity. The lateral structure of the emitters is a tapered ridge with a narrow angle $\left(<1^{\circ}\right)$ and an overall cavity length of $2.5 \mathrm{~mm}$ [5]. The tapered design allows high power emission together with single transverse mode operation thanks to the widened active section. The far field full-width at $1 / \mathrm{e}^{2}$ in the slow axis is $2.7^{\circ}$ and the $\mathrm{M}^{2}$ parameter is $<2$. The rear facet is high-reflection coated. Thanks to the antireflection coating $\left(\mathrm{R}<10^{-3}\right)$ on the front facet of the laser array, no laser emission but only amplified spontaneous emission is observed without external cavity.

Our experimental setup is shown in Figure 2. The beam from the laser array is collimated in the fast axis direction with a high NA acylindric lens. A volume Bragg grating from ONDAX, Inc is used as the external mirror. It is designed to reflect $40 \%$ at $976 \mathrm{~nm}$ with a spectral bandwidth $\Delta \lambda=0.3 \mathrm{~nm}$. The angular acceptance of the volume Bragg grating (VBG) is $\Delta \theta=2^{\circ}$ (FWHM), which is larger than the divergence of the laser array. The diffraction efficiency of the VBG will then remain close to maximum. The pitch between the emitters being $100 \mu \mathrm{m}$, the Talbot self-imaging length is $20.4 \mathrm{~mm}$, and the VBG is located at a quarter of the Talbot distance from the front facet. This setup results in a very compact and simple cavity with only one intracavity element for limited intracavity losses. The whole setup is mounted on a copper bloc and actively thermally regulated.

With the VBG as the output coupler of the cavity, a power of $1.7 \mathrm{~W}$ at an operating current of $3.9 \mathrm{~A}$ has been obtained. The slope efficiency is $0.6 \mathrm{~W} \cdot \mathrm{A}^{-1}$ (see figure 4). With unslanted VBG, the cavity operates in the out-ofphase mode, but by tilting the VBG by an angle $\alpha=\lambda / 2 p$, the in-phase mode is selected. The slow-axis angular distribution of the laser was measured with a CCD camera at the Fourier plane of a cylindrical collimating lens. The far-field profile exhibits interferences peaks, which is a clear evidence of the phase locking in the in-phase mode. A fringe contrast as high as $80 \%$ as been measured at low power $(800 \mathrm{~mW}, \mathrm{I}=2.5 \mathrm{~A})$, which demonstrates the high degree of coherence induced by the Talbot external cavity. At higher currents, the contrast decreases to $70 \%$ due to increased incoherent free-running contribution. The maximal output power is only limited by the thermal roll-over of the array which appears at $4 \mathrm{~A}$ under our experimental conditions. We have checked that similar performance is obtained with a plane mirror as the output coupler instead of the VBG; the coherent combining is thus not degraded by the use of a volume grating.

In order to characterize the spectrum of the laser array, we have measured the so-called spectrally resolved near field (SNeaF) of the laser array. The near-field spatial profile is imaged on the input slit of a monochromator, and a camera is positioned in the output plane. A typical SNeaF of our external-cavity laser is given in Figure 5: the slowaxis near-field profile of the bar is displayed in the vertical direction, and the spectrum of each separate emitter in the horizontal one. Obviously each emitter is locked at the Bragg wavelength, with no evidence of parasitic lines. A 
high-resolution $(10 \mathrm{pm})$ spectrum is also given in Figure 6, which confirms the strong spectral locking induced by the VBG; no relative emitters drift is observed, and the whole bar spectrum remains within 100 pm (FWHM).

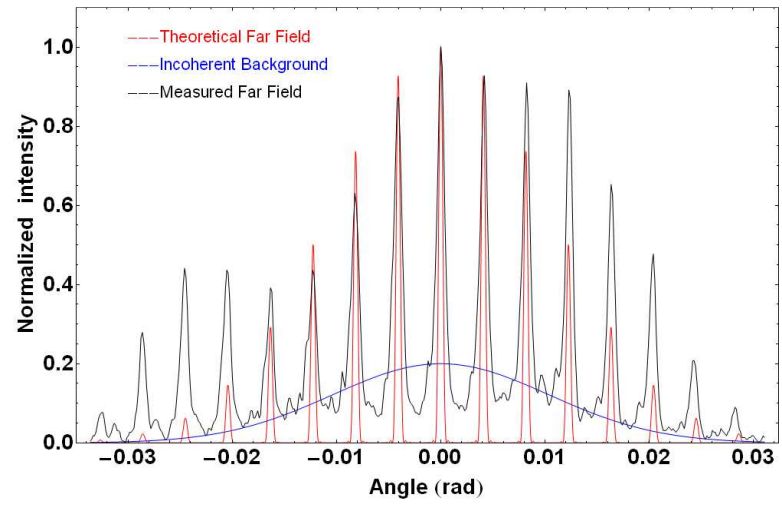

Fig. 3. Far field profile at $\mathrm{I}=3.9 \mathrm{~A}(\mathrm{P}=1.7 \mathrm{~W})$. (black line - measured far field, red line - theoretical far field, blue line - incoherent freerunning contribution)

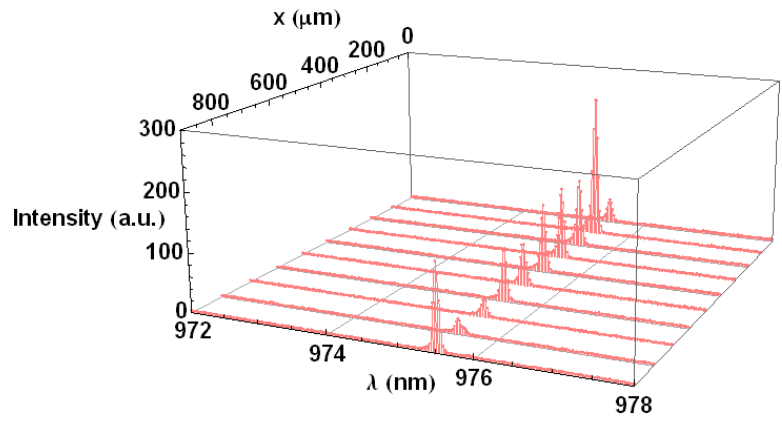

Fig. 5. Spectrally resolved near-field of the cavity $(\mathrm{I}=4 \mathrm{~A}, \mathrm{P}=1.7 \mathrm{~W})$

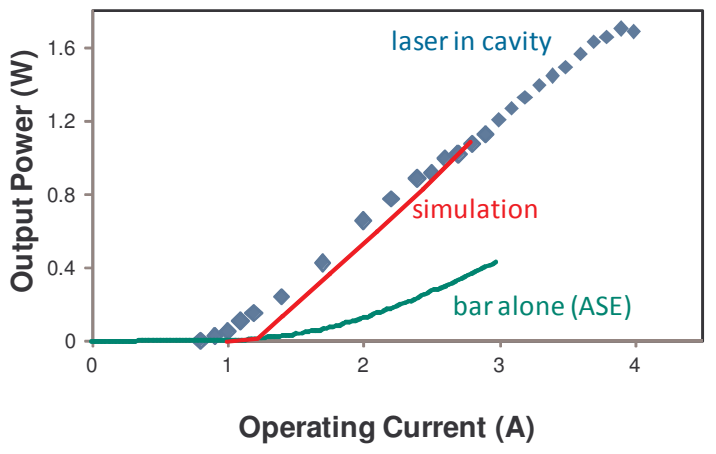

Fig. 4. L-I characteristic ( green - laser array alone, blue - laser in external cavity, red - simulation )

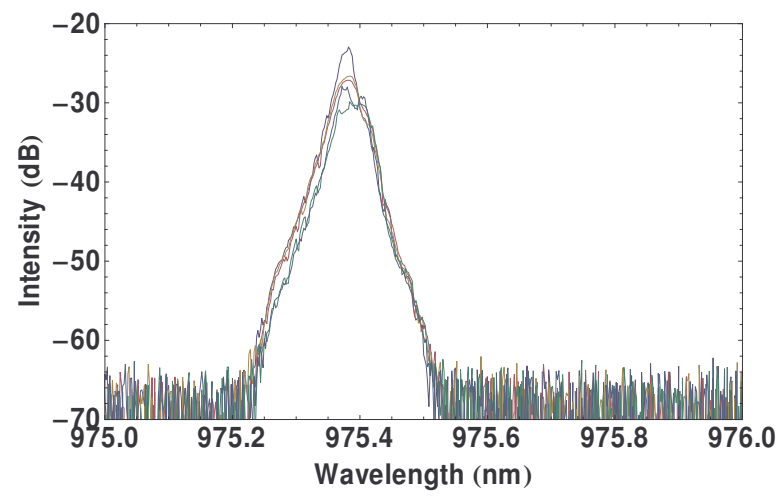

Fig. 6. Superimposed spectra of 5 of the 10 emitters, measured with a 10 -pm resolution optical spectrum analyser $(\mathrm{I}=2.8 \mathrm{~A}, \mathrm{P}=1 \mathrm{~W})$

\section{Conclusion}

We have modeled the external-cavity operation of an array of index-guided tapered lasers, by use of an iterative method inside the semiconductor waveguides and in the free-space external cavity. We have demonstrated both theoretically and experimentally the efficient coherent combining of an array of tapered lasers in a Talbot external cavity. Furthermore wavelength stabilization has been achieved simultaneously by use of a volume Bragg grating as the external mirror. A very simple and compact cavity has been developed. A high coherence is obtained with a contrast higher than 0.7. The spectrum is narrowed to $100 \mathrm{pm}$ and stabilized at the Bragg wavelength whatever the operating conditions. This results in a high brightness and high spectral quality laser source. Further increase of the output power is expected from a lower grating reflectivity.

Acknowledgments: This work was supported within the European project BRIGHTER.EU under grant referenced FP6-IST-035266. D. Paboeuf thanks the French Ministry of Defense (Délégation Générale de l'Armement) for funding his $\mathrm{PhD}$.

\section{References}

[1] A.F. Glova et al., "Phase-locking of optically coupled laser", Quantum Electronics 33 (4), 283-306 (2003)

[2] B. L. Volodin et al., "Wavelength stabilization and spectrum narrowing of high-power multimode laser diodes and arrays by use of volume Bragg gratings," Opt. Lett. 29, 1891-1893 (2004)

[3] J.W. Goodman, "Introduction to Fourier Optics" (Roberts \& Company 2005), Chap. 4.

[4] J.J. Lim et al.,’Design of Wide-Emitter Single-Mode Laser Diodes”, IEEE J. Quant. Elec. 41 (4) 506-516 (2005)

[5] M. Krakowski et al., "1 W high brightness index guided tapered laser at $980 \mathrm{~nm}$ using Al-free active region materials," Electron. Lett. 39, 1122-1123 (2003). 